\title{
Fluorescein angiography of anterior uveal melanocytic tumours
}

\author{
J K DART,' R J MARSH, ${ }^{23}$ A GARNER,' AND R J COOLING ${ }^{3}$ \\ From the 'Institute of Ophthalmology, University of London, the ${ }^{2}$ Western Ophthalmic Hospital, and \\ ${ }^{3}$ Moorfields Eye Hospital, London
}

SUMMARY A retrospective analysis of 32 cases of anterior uveal melanocytic tumours included 14 cases in which the tumours could be categorised histologically as malignant, of intermediate cytology with equivocal features of malignancy, or as benign progressive naevi. An additional eight cases without a histological diagnosis were included as benign naevi, because there had been no change in their clinical characteristics after a minimum follow-up of four years. Clinical features and iris fluorescein angiographic (IFA) findings were analysed with respect to these groups in an attempt to identify features predictive of malignant or locally invasive behaviour. Very small tumours were more likely to be benign $(p=0 \cdot 029)$. Glaucoma and episcleral vascular dilatation occurred in ciliary body tumours only. Tumours involving the anterior chamber angle proved more likely to be malignant $(\mathrm{p}=0 \cdot 019)$. IFA showed a disorganised vasculature and gross late leakage in $4 / 7(56 \%)$ malignant melanomas as well as in $2 / 6(33 \%)$ tumours with intermediate cytology. No benign tumours showed these features. Four out of nine $(44 \%)$ benign tumours, but no tumours with malignant or intermediate cytology, showed complete masking of fluorescence. Early leakage of dye from tumour vessels and a geometric tumour vasculature were not specific features of any tumour category. IFA correlated with the cytology and behaviour of anterior uveal melanocytic tumours in $11 / 22(50 \%)$ of our cases.

Melanocytic tumours are the commonest that affect the iris. They may behave in three ways. Most are slow growing or static. Some are locally invasive, with benign cytological characteristics, and may be regarded as benign progressive naevi even though local growth may result in the loss of the eye.' Lastly they may metastasise, though this is rare, occurring in $3-5 \%$ of cases. ${ }^{12}$ Ciliary body lesions encroaching on the iris have a worse prognosis and are sometimes impossible to differentiate clinically from a primary iris tumour. Clinical features apart from rapid growth are of little value in predicting the behaviour or cytological characteristics of a lesion. ${ }^{13}$ Excision biopsy carries appreciable morbidity in eyes which are generally asymptomatic with good sight. ' A noninvasive investigation that might predict tumour behaviour and cytology would assist in the management of these lesions.

It has been suggested that iris fluorescein angiography (IFA) is capable of differentiating between

Correspondence to J K G Dart, FRCS, Department of Clinical Ophthalmology, Moorfields Eye Hospital, City Road, London EC1V 2PD. tumours with and without metastatic potential. ${ }^{34}$ Fluorescein angiography allows assessment of the degree of abnormality of the iris tumour vasculature as well as secondary effects of tumour growth on surrounding tissues. It is assumed that abnormal patterns of tumour vasculature and permeability of iris tumour vessels reflect the degree of differentiation and cytological abnormality of the tissues. The pathophysiological associations of these angiographic features have been discussed elsewhere. ${ }^{34}$

There has been general agreement in the previous literature on the interpretation of certain patterns of IFA in iris melanocytic tumours. Masking of fluorescence by the lesion has been considered by all authors who have described it as indicative of a benign lesion. ${ }^{37}$ The presence of a geometric, organised (leaf-like, ${ }^{6}$ network, ${ }^{3}$ filigree ${ }^{4}$ ) vascular pattern has been associated with benign lesions by some ${ }^{46}$ and by others as potentially malignant. ${ }^{3}$ Disorganised irregular tumour vasculature has similarly been considered to denote malignancy. ${ }^{45}$ The importance of leakage into and around the tumour is open to dispute. Some have regarded it as indicative of 
malignancy, especially if early or profuse,,$^{3+6}$ but most have considered it associated with both benign and malignant tumours. ${ }^{3-5}$ Areas of leakage at sites remote from the tumour such as the pupil margin are probably of no significance. ${ }^{3+}$

Unfortunately most of the earlier reports are difficult to evaluate because of doubt about the histopathological criteria for a diagnosis of malignancy. ${ }^{1}$ Recently Jakobiec et al. ${ }^{4}$ evaluated the cytological characteristics and described the fluorescein appearance of a series of iris melanocytic tumours, but, as they included only two cases that were considered to be malignant, reservations have to be made about their interpretation of IFA patterns. Tumours of uncertain or intermediate characteristics have been difficult to categorise by any system of fluorescein or pathological classification, further complicating the analysis of data in previous studies.

In the past 10 years we have encountered 32 cases of melanocytic iris tumour, 14 of which have been excised and evaluated histologically with reference to the nine group classification of Jakobiec and Silbert.' A simple method for describing IFA patterns was used and the fluorescein characteristics correlated with the histopathological classification and behavioural characteristics of the tumours. These results were analysed to determine which fluorescein parameters were most helpful in indicating the likely cytological composition of the tumours and their future behaviour.

\section{Patients and methods}

Thirty-two cases were initially referred to one of us (RJM) for clinical evaluation and IFA. Twenty-one cases have been reviewed in the last year: 11 cases have been reviewed with repeat photography and angiography, 10 have been followed up by a review of the case notes supplied by the referring practitioner, and three have died. Eight cases were lost to followup.

Examination. Each patient had a full ophthalmological examination with slit-lamp microscopy, gonioscopy, applanation tonometry, and funduscopy. IFA was performed with a Zeiss motorised slitlamp camera with an accessory halogen illuminator and a $16 \mathrm{~mm}$ cine camera as previously described. ${ }^{\mathrm{N}}$ Photographs were taken 7 seconds after injection of $5 \mathrm{ml}$ of $20 \%$ fluorescein and repeated every 1.5 seconds with late pictures at 3 minutes.

Interpretation of iris angiograms. The IFAs were reviewed in each case and classified by a simple system in which each of four possible tumour angiographic features was assessed and graded separately. These were the degree of masking, the timing and degree of any leakage from the tumour vessels, the structure of the tumour vasculature, and the phase of filling of the tumour vessels.

Pathology. Excision biopsies were all examined by one of us (AG) using light microscopy and, in selected cases, electron microscopy. The tumours were classified according to the nine group classification of Jakobiec and Silbert ${ }^{1}$ (J\&S 1-9). For simplicity the classification was reduced to three groups: malignant melanomas (J\&S 7, 8, 9), intermediate melanocytic tumours (J\&S 5,6), and benign or progressive naevi (J\&S $1,2,3,4)$. Lesions included in the intermediate group presented cytological features which did not allow clear categorisation as benign or malignant. It encompassed tumours that could be classified histologically as spindle cell naevus with surface plaque (J\&S 5) and borderline spindle cell naevus (J\&S 6). Unlike Jakobiec and Silbert, ' we considered that these cytological features

Table 1 Classification of anterior uveal tumours

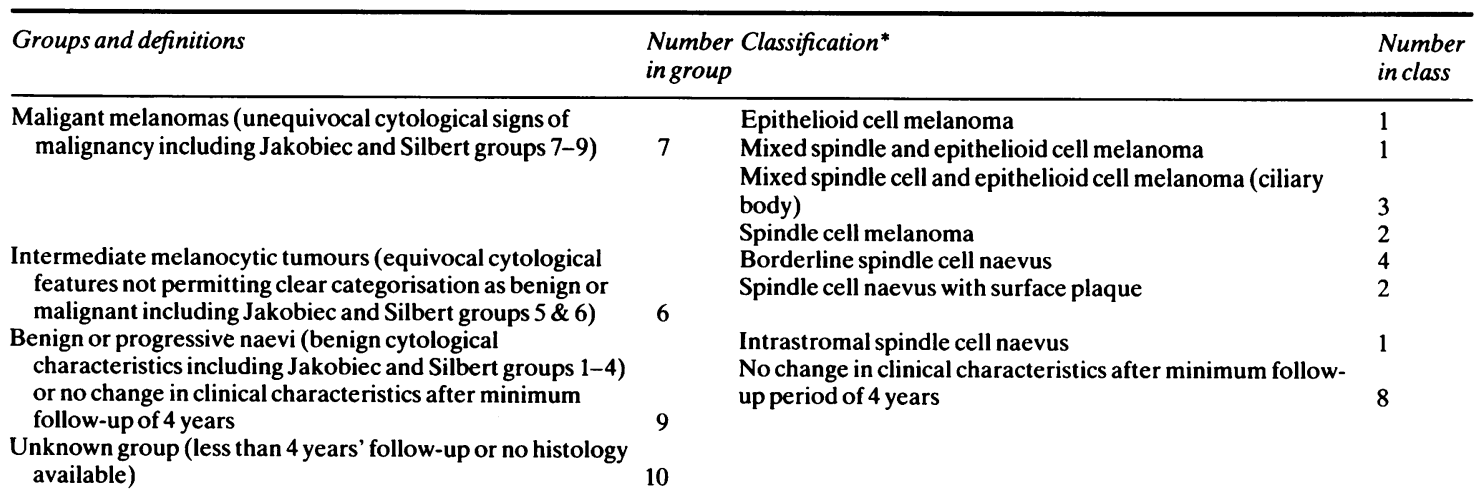

*The histological classification followed that of Jakobiec and Silbert. All histological diagnoses were for primary iris tumours except for the three ciliary body tumours involving iris. 
Table 2 Age and sex distribution and follow-up period and deaths in patients with anterior uveal tumours

\begin{tabular}{|c|c|c|c|c|c|c|}
\hline & \multicolumn{5}{|l|}{ Tumour group } & \multirow[t]{2}{*}{ Total } \\
\hline & Malignant iris & $\begin{array}{l}\text { Malignant ciliary } \\
\text { body }\end{array}$ & Intermediate & Benign & Unknown & \\
\hline Age: Mean & 35 & 48 & 47 & 70 & 51 & 53 \\
\hline Range & $25-46$ & $43-54$ & $27-59$ & $56-81$ & $22-84$ & $22-84$ \\
\hline SD & 8.6 & $5 \cdot 5$ & 10.9 & $8 \cdot 5$ & & \\
\hline Sex: $M / F$ ratio & 4:0 & $1: 2$ & $4: 2$ & $3: 6$ & $7: 4$ & $19: 13$ \\
\hline \multicolumn{7}{|c|}{ Follow-up period in months: } \\
\hline Mean & 65 & 25 & 27 & 80 & 16 & 43 \\
\hline Range & $1-97$ & $14 \cdot 5-51$ & $6-67$ & $51-144$ & $1-60$ & $1-144$ \\
\hline
\end{tabular}

ANOVA on age distribution in malignant, intermediate, and benign tumour groups. F statistic $16 \cdot 89 ; p=<0 \cdot 001$.

could not be regarded as unequivocal evidence of benignity. Three ciliary body tumours have been included as these presented clinically as peripheral iris tumours.

The remaining tumours without histology were each allocated to one of two groups on the basis of their clinical and behavioural characteristicsbenign naevus or unknown. The benign naevus group included lesions that had been observed for a minimum of four years without change. The unknown group included all the remaining tumours in which there was either no histological diagnosis, evidence of changing clinical characteristics, or a follow-up period of less than four years.

Statistical methods. Analysis of variance (ANOVA) was used to analyse the differences in the age distribution of subjects in the different tumour groups and the $\chi^{2}$ test to analyse the proportional data when appropriate.

\section{Results}

Table 1 shows the distribution of tumours into the four groups: malignant melanomas (malignant group), intermediate melanocytic tumours (intermediate group), benign or progressive naevi (benign group), and unknown. The age and sex characteristics and the follow up periods are shown in Table 2. ANOVA shows that there are significant differences in the age distribution for the different tumour groups $(p=<0 \cdot 001)$. Table 3 shows the length of history together with the number of patients in each group in whom the tumour was discovered at an ophthalmic examination for refraction or symptoms unrelated to the tumour. It can be seen that neither the length of history nor the discovery of an asymptomatic tumour on routine examination is related to the eventual outcome. Table 3 also shows the surgical management and deaths. Of the three patients who died one had a malignant ciliary body tumour and had a presumptive diagnosis of metastatic disease related to this. Unfortunately no post-mortem examination was carried out to confirm the cause of death. Of the remaining two, both in the unknown group, one died of metastatic disease for which no primary tumour was identified and once again no

Table 3 History

\begin{tabular}{|c|c|c|c|c|c|c|}
\hline \multirow[b]{3}{*}{ Length of $<1$} & \multicolumn{5}{|l|}{ Tumourgroup } & \multirow[t]{2}{*}{ Total } \\
\hline & Malignant iris & $\begin{array}{l}\text { Malignant ciliary } \\
\text { body }\end{array}$ & Intermediate & Benign & Unknown & \\
\hline & & & 3 & & 1 & 4 \\
\hline history 1-2 & & 2 & 1 & 1 & 2 & 6 \\
\hline in years $\quad 3-4$ & & 1 & & 2 & & 3 \\
\hline $5-10$ & 3 & & 1 & 4 & 2 & 10 \\
\hline $10-20$ & & & & 3 & 2 & 5 \\
\hline$>20$ & & & 1 & 1 & & 2 \\
\hline Life & 1 & & & 1 & & 2 \\
\hline Discovered on routine & & & & & & \\
\hline $\begin{array}{l}\text { examination } \\
\text { Surgical management }\end{array}$ & 1 & 1 & 3 & 8 & 5 & 18 \\
\hline Iridectomy & 3 & & 5 & 1 & & 9 \\
\hline Iridocyclectomy & 1 & & 1 & & & 2 \\
\hline Enucleation & & 3 & & & & \\
\hline Deaths & & 1 Metastatic & & & $\begin{array}{l}1 \text { Metastat } \\
1 \text { Heart dis }\end{array}$ & ry uncertain) \\
\hline
\end{tabular}


Table 4 Clinical features in relation to tumour classification

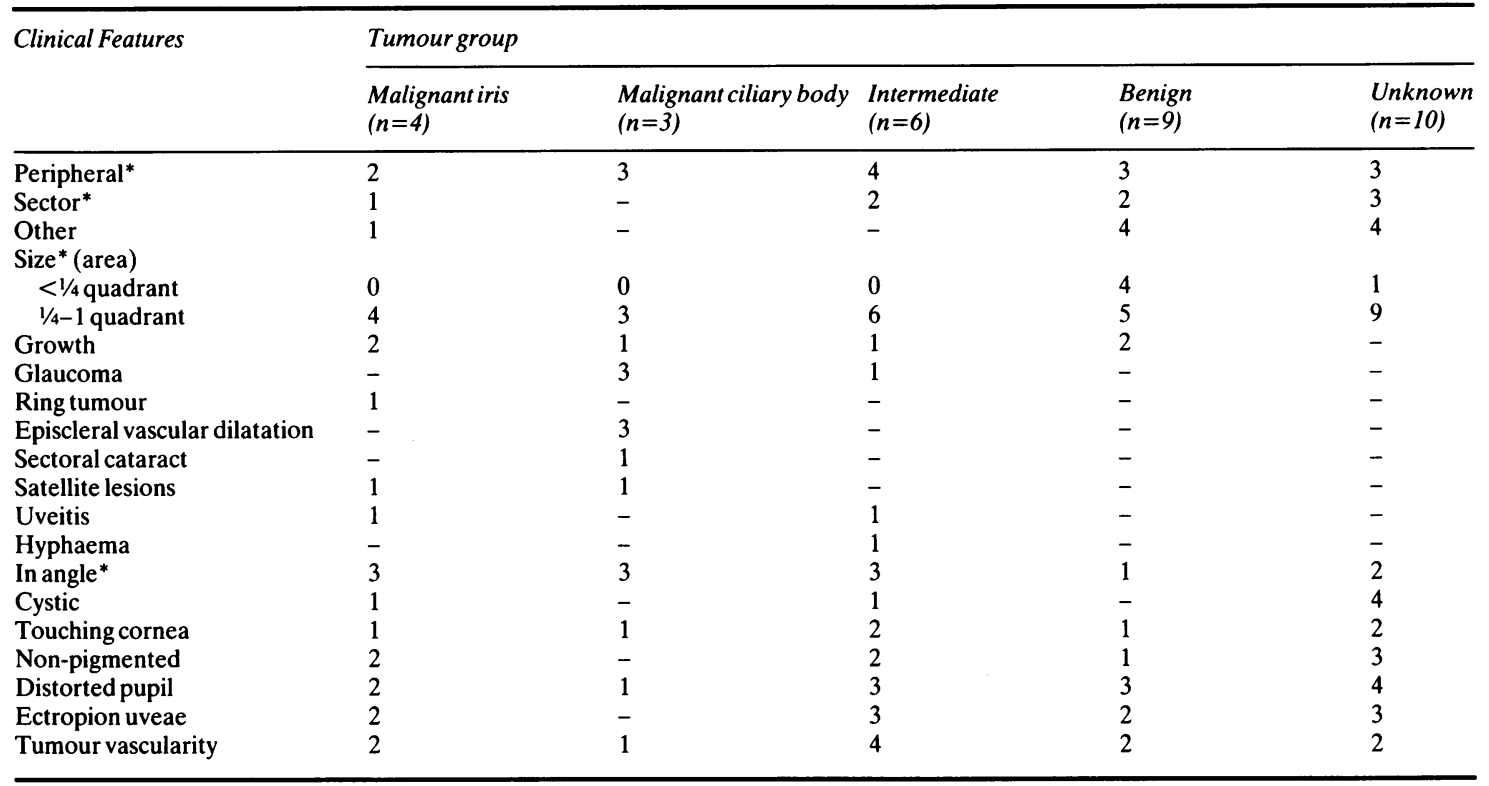

${ }^{*}$ The frequency of these features was tested by the $\chi^{2}$ test in the malignant, intermediate, and benign groups.

The only significant values at the $5 \%$ level were for tumours of less than $1 / 4$ quadrant in area, which occurred only in the benign group $(p=0.029)$, and the presence of tumour in the angle $(p=0.019)$, which was more frequent in the malignant and intermediate groups.

post-mortem study was performed. The remaining death was from unrelated cardiac disease. The incidence of prominent clinical features in each group is shown in Table 4. In this series growth was recorded in all tumour categories, including tumours of two patients in the benign group who reported slow growth over many years that did not continue during the follow up period of 5-7 years. Glaucoma and episcleral vascular dilatation were features of ciliary body tumours but were not identified in the iris tumours in this series. Some of these clinical features were sufficiently common to allow statistical analysis by the $\chi^{2}$ test to compare their frequency in the different groups; these are asterisked in Table 4. Because none of the tumours in this series exceeded one quadrant in area, the effect of size was difficult to quantify, though very small tumours, involving less than 1 quarter of a quadrant, were more likely to be benign than larger tumours $(p=0 \cdot 029)$. Moreover tumours involving the angle were more often malignant than benign $(p=0 \cdot 019)$. As tumours of all sizes involved the angle, this finding is probably independent of size, but numbers were not large enough for statistical analysis. Except for these findings the different behavioural and histopathological groups could not be distinguished on the basis of clinical features.

The IFA characteristics and their occurrence in the different tumour groups are shown in Table 5. Five of these characteristics were selected for correlation with the three tumour groups composed of the 22 cases for which the tumour cytology and behaviour were known. They were disorganised vascular pattern, early leakage, a gross late degree of leakage, complete masking ('angiographic silence'), and geometric vascular pattern. These findings are summarised in Table 6, which shows that disorganised vascular pattern and gross late leakage did not occur in the benign group at all. However, they were not common in either the malignant or intermediate group: disorganised vascular pattern occurring in only $4 / 7(57 \%)$ of the malignant tumours (Figs. $1 \mathrm{~A}-\mathrm{C})$, and in $2 / 6(33 \%)$ of the intermediate group (Figs. 2A-C), while early leakage was found in only $2 / 7(29 \%)$ of malignant tumours (Figs. 3A-E), 2/6 intermediate group tumours (Figs. 4A-C) and 1/9 tumours in the benign group (Figs. 5A, B). One or more of these three IFA characteristics were present in $6 / 7(86 \%)$ of malignant tumours. The same was true of $3 / 6(50 \%)$ of the intermediate group and one case in the benign category, a tumour that had been followed up without change for eight years. Early leakage was not specific for tumours of malignant or intermediate cytology. Gross late leakage was uncommon.

Complete masking was only found in $4 / 9(44 \%)$ of the benign group (Figs. 6A, B). Geometric vascular pattern occurred in all groups (Figs. 5, 7A-E, 8A, B) 
Table 5 Classification system for IFAs and number of tumours in each IFA class

\begin{tabular}{|c|c|c|c|c|c|}
\hline \multirow{2}{*}{$\begin{array}{l}\text { Fluorescein characteristic } \\
\text { Each assessed and graded } \\
\text { separately }\end{array}$} & \multicolumn{5}{|l|}{ Tumourgroup } \\
\hline & Malignantiris & Malignant ciliary body & Intermediate & Benign & Unknown \\
\hline \multicolumn{6}{|l|}{ Masking: } \\
\hline Complete & - & - & - & 4 & 3 \\
\hline Incomplete & 3 & 3 & 2 & 5 & - \\
\hline None & - & - & 4 & - & - \\
\hline \multicolumn{6}{|l|}{ Leakage: } \\
\hline None & - & - & - & - & - \\
\hline Early moderate* & 1 & 1 & 2 & 1 & 1 \\
\hline Early gross & - & - & - & - & - \\
\hline Late moderate $\dagger$ & 3 & 1 & 5 & 4 & 7 \\
\hline Late gross $\ddagger$ & - & - & 1 & - & - \\
\hline \multicolumn{6}{|l|}{ Filling pattern: } \\
\hline \multicolumn{6}{|l|}{ Geometric with vessels of } \\
\hline \multicolumn{6}{|l|}{ Disorganised with vessels of } \\
\hline varying calibre & 2 & 2 & 2 & - & - \\
\hline Dilated radial vessels & - & 2 & 1 & - & 2 \\
\hline \multicolumn{6}{|l|}{ Phase of tumour filling: } \\
\hline In phase with iris & 3 & 2 & 5 & 1 & 5 \\
\hline Out of phase with iris & 1 & 1 & 1 & 2 & 1 \\
\hline Different phases within tumour & $r-$ & - & - & - & - \\
\hline $\begin{array}{l}\text { Patients having repeat } \\
\text { angiography }\end{array}$ & 2 & 1 & 1 & 5 & 2 \\
\hline
\end{tabular}

${ }^{*}$ Early, within 10 seconds of appearance of dye in tumour. $\nmid$ Late, 10 seconds or more after appearance of dye in tumour. $\ddagger$ Dye obscuring all detail of tumour.

except the malignant ciliary body melanomas and consequently appears to be non-specific for tumour cytology or behaviour.

The $\chi^{2}$ test was used to compare the frequency of each feature in the different tumour groups. No significant difference could be identified between intermediate melanocytic tumours and either benign naevi or malignant melanomas for any individual fluorescein characteristic, though the presence of a disorganised vascular pattern did not occur in the benign group $(p=0.055)$. A higher proportion of tumours in the malignant and intermediate groups compared with the benign group showed one or more of the characteristics that others have previously regarded as being indicators of malignancy $(\mathrm{p}=0.019)$.

Table 6 Analysis of fluorescein characteristics expected to be specific indicators of tumour cytology and behaviour

\begin{tabular}{|c|c|c|c|c|}
\hline \multirow[t]{2}{*}{ Fluorescein characteristics } & \multicolumn{4}{|l|}{ Tumour group } \\
\hline & Malignant iris & $\begin{array}{l}\text { Intermediate } \\
n=6\end{array}$ & $\begin{array}{l}\text { Benign } \\
n=9\end{array}$ & $\chi^{2}$ test \\
\hline \multicolumn{5}{|c|}{ Fluorescein characteristics indicative of malignant melanomas } \\
\hline Disorganised vascular pattern & & $2(33 \%)$ & - & $\begin{aligned} \chi^{2} & =5.170 \\
p & =0.055\end{aligned}$ \\
\hline Early leakage & & $2(33 \%)$ & $1(11 \%)$ & $\begin{aligned} \chi^{2} & =1.353 \\
p & =0.508\end{aligned}$ \\
\hline Gross late leakage & & $1(17 \%)$ & - & $\begin{aligned} \chi^{2} & =1.419 \\
p & =0.492\end{aligned}$ \\
\hline Any of above & & $3(50 \%)$ & $1(11 \%)$ & $\begin{aligned} \chi^{2} & =7.916 \\
p & =0.019\end{aligned}$ \\
\hline \multicolumn{5}{|c|}{ Fluorescein characteristics indicative of benign naevus } \\
\hline Complete masking & & - & $4(44 \%)$ & $\begin{aligned} \chi^{2} & =5 \cdot 140 \\
p & =0.077\end{aligned}$ \\
\hline Geometric vasculature & & $4(66 \%)$ & $3(33 \%)$ & $\begin{aligned} \chi^{2} & =5.611 \\
p & =0.060\end{aligned}$ \\
\hline Any of above & & $4(66 \%)$ & $7(78 \%)$ & $\begin{aligned} \chi^{2} & =3 \cdot 650 \\
p & =0 \cdot 161\end{aligned}$ \\
\hline
\end{tabular}




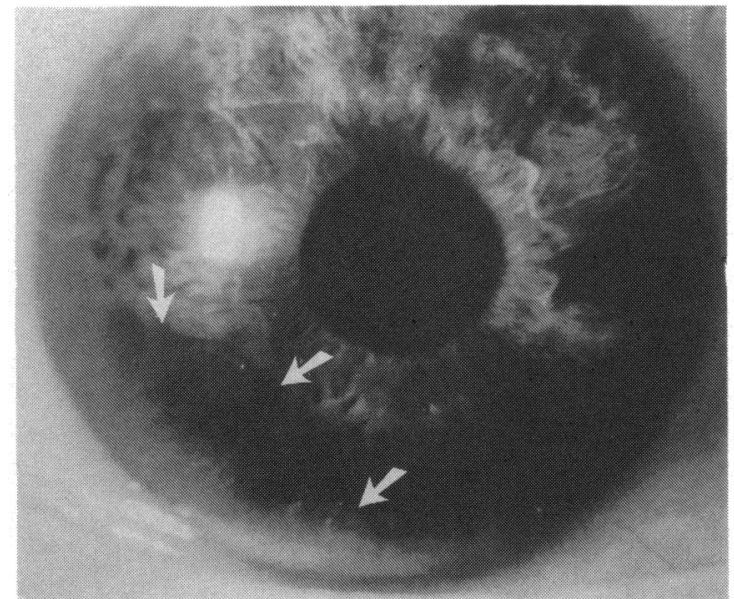

Fig. 1 Case 17. Mixed spindle cell and epithelioid melanoma (ciliary body). In this and subsequent clinical photographs the arrows point to the tumours. A: Clinical appearance of a tumour, involving the angle, that was deeply pigmented and markedly vascular. Associated findings were iris freckles and heterochromia, pigment in the angle, and $a$ distorted pupil.

Follow-up. Angiography was repeated in 11 cases. No change in the fluorescein characteristics was noted in any case apart from an increase in the size of some lesions (Fig. 7). In three instances when tumours were excised and found to be cytologically malignant there had been evidence of growth on the angiogram. In one case postoperative angiography confirmed the presence of vascularised metastases that had seeded on to the surface of the iris when the tumour fragmented at the time of surgery (Fig. 3).

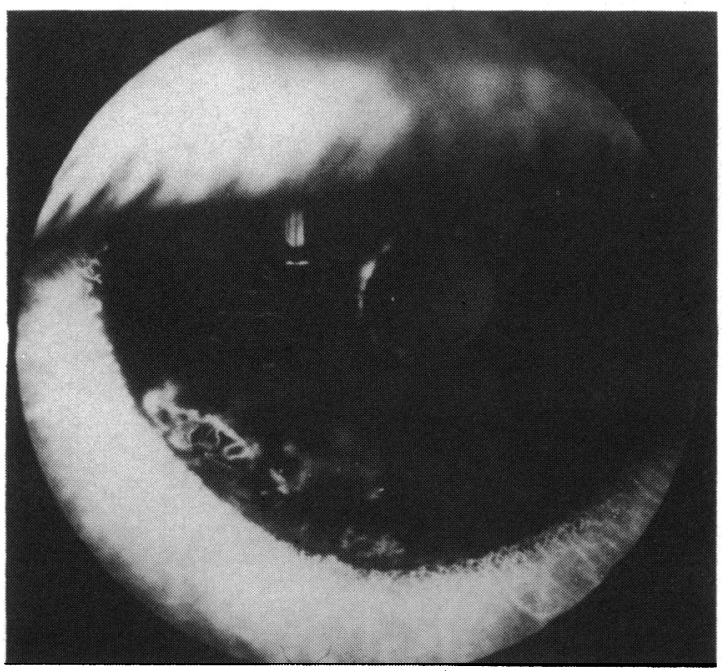

Fig. 1B IFA showing a disorganised vascular pattern filling out of phase with the iris vessels.

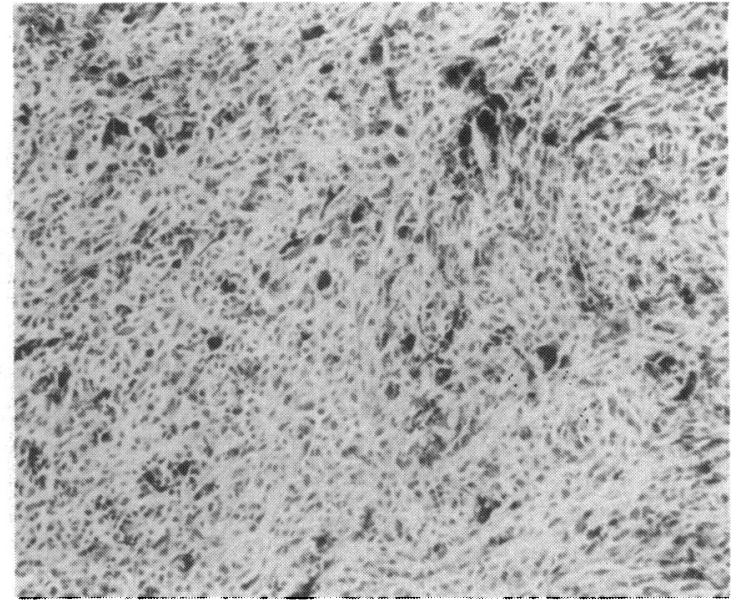

Fig. 1C The tumour is composed of both spindle and epithelioid cells. Hand $E, \times 115$.

\section{Discussion}

There is no reliable clinical method of predicting tumour behaviour apart from observation of rapid growth involving the surrounding tissues or frank metastasis. Cytological features are a recognised way of determining whether a tumour may have the potential to display this behaviour. Unfortunately the criteria are not sufficiently precise or constant to permit unfailing accuracy in the diagnosis of anterior uveal melanocytic tumours. ${ }^{129}$ However, it has been suggested that IFA findings reflect both tumour behaviour and cytology, ${ }^{3-7}$ although here again the criteria are open to dispute. Difficulty in the interpretation of fluorescein findings has been complicated for some cases, in which biopsies were undertaken after IFA, by doubt about the basis of the cytological evaluation. ${ }^{4}$ This arose because the descriptions of the tumour histology were incomplete. Although the prognostic inferences drawn by Jacobiec and Silbert ${ }^{\prime}$ from their analysis of the histological features of a series of iris melanomas have been criticised, ${ }^{9}$ their scheme still provides a good basis for describing the morphology of these tumours. Its chief merit is that it provides sufficient detail for reasonably precise categorisation of individual biopsy specimens. We have used these histological categories in our retrospective analysis of 32 cases of anterior uveal melanocytic tumours which provided 22 cases in which the behaviour and cytology of the lesions could be used to clearly categorise them into three groupsmalignant, intermediate, and benign. This has enabled analysis of clinical and fluorescein features to establish whether any of these are reliable predictors of these categories of tumour.

In this series it is interesting that the patients with 


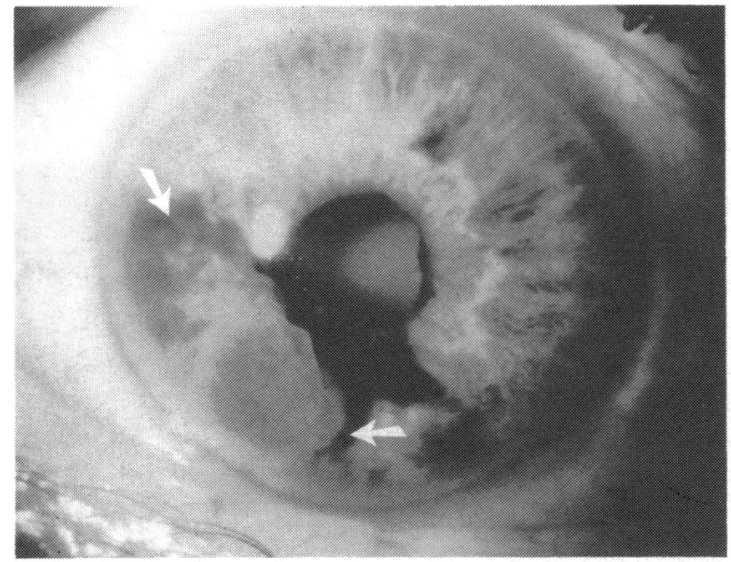

Fig. 2 Case 11. Spindle cell naevus with surface plaque (iris). A: Clinical appearance showing an elevated lesion with marked ectropion uveae.

tumours in the malignant groups were younger than those with tumours showing benign characteristics $(p<0.001)$. This would not be expected on the hypothesis that most tumours displaying malignant cytological features have transformed from benign lesions."

Apart from the observation of rapid growth, clinical features are of little value in the prediction of tumour type. ${ }^{134}$ Analysis of this small series confirmed that glaucoma, episcleral vascular dilatation, and sectoral cataract are features of ciliary body tumours, which are more commonly malignant than iris tumours. However, it is evident from a larger series that these features do not of themselves imply malignancy.' The only significant clinical findings

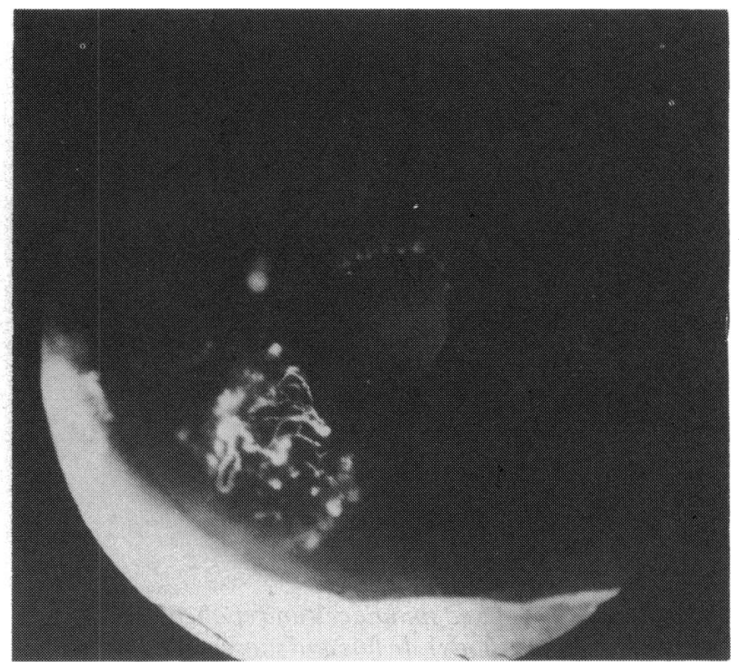

Fig. 2B IFA showing incomplete masking and a disorganised vascular pattern.

were that very small tumours, of less than $1 / 4$ quadrant in area, were more likely to be benign $(p=0.029)$ and that tumours involving the angle were more likely to be malignant $(p=0 \cdot 019)$. Because none of the tumours in this series exceeded 1 quadrant in area the effect of size was difficult to quantify. As angle involvement occurred in tumours of all sizes, the association of malignancy with tumour involving the angle is likely to be independent of size.

We found difficulty in interpreting our findings in the light of the previous reports because of two factors. Fluorescein angiograms could not neatly be fitted into the patterns described by Demeler, ${ }^{3}$
Fig. 2C Histologyshows a well demarcated plaque of small spindle cells protruding from the anterior surface of the iris. Note the prominent blood vessels. $H$ and $E$, $\times 127$.

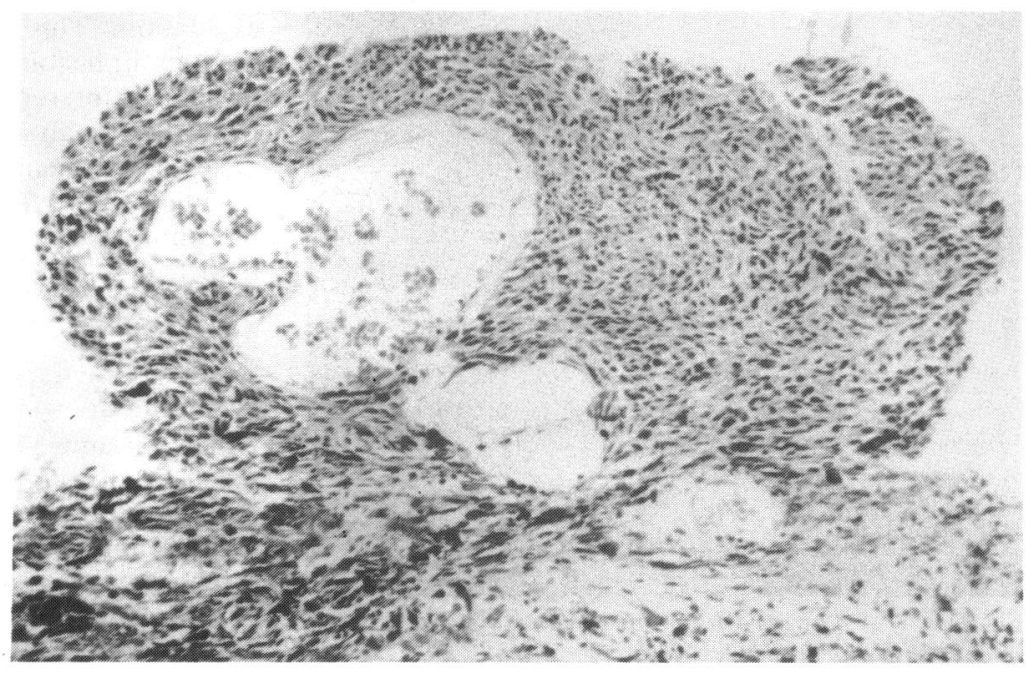




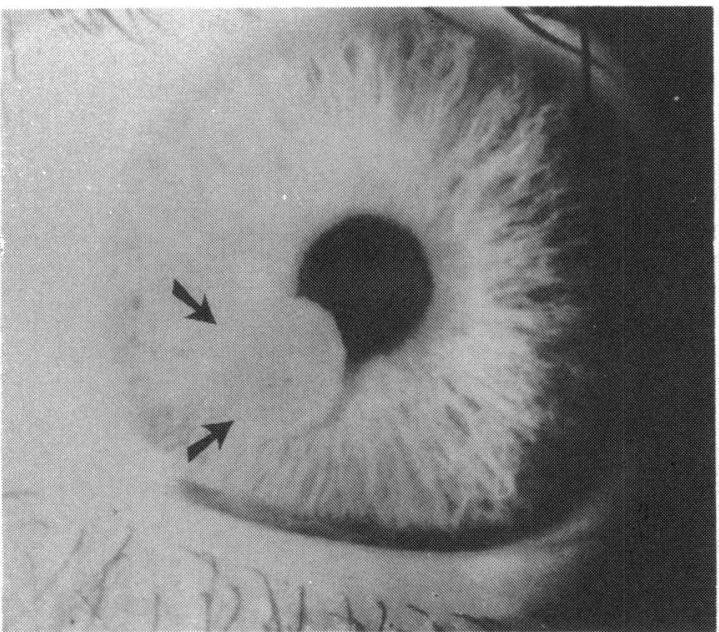

Fig. 3 Case 18. Mixed spindle cell and epithelioid melanoma (iris). A: Clinical appearance of a vascular nonpigmented tumour associated with a distorted pupil and ectropion uveae.

Jakobiec et al. ${ }^{+}$and Brovkina and Chichua, ${ }^{\circ}$ though Kottow's 'sas comparable. Secondly, the criteria for the histopathological diagnosis of iris malignant melanomas have not been generally agreed; this problem has been fully discussed by Jakobiec et al. ${ }^{1}$

Our classification of IFA is simple, reasonably

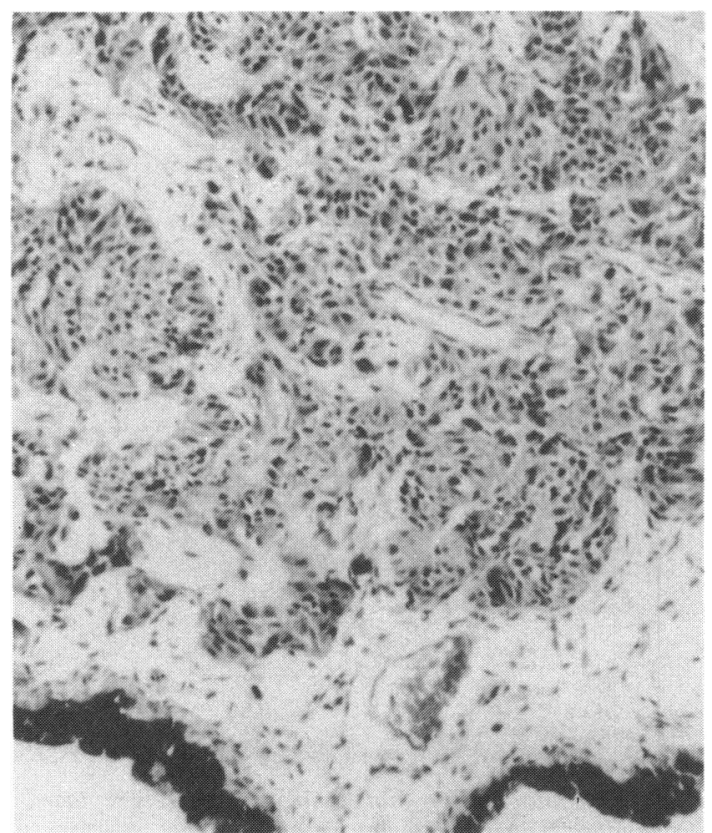

Fig. 3C Histology shows that the iris stroma is infiltrated by a mixture of epithelioid and spindle cells. $H$ and $E, \times 136$.

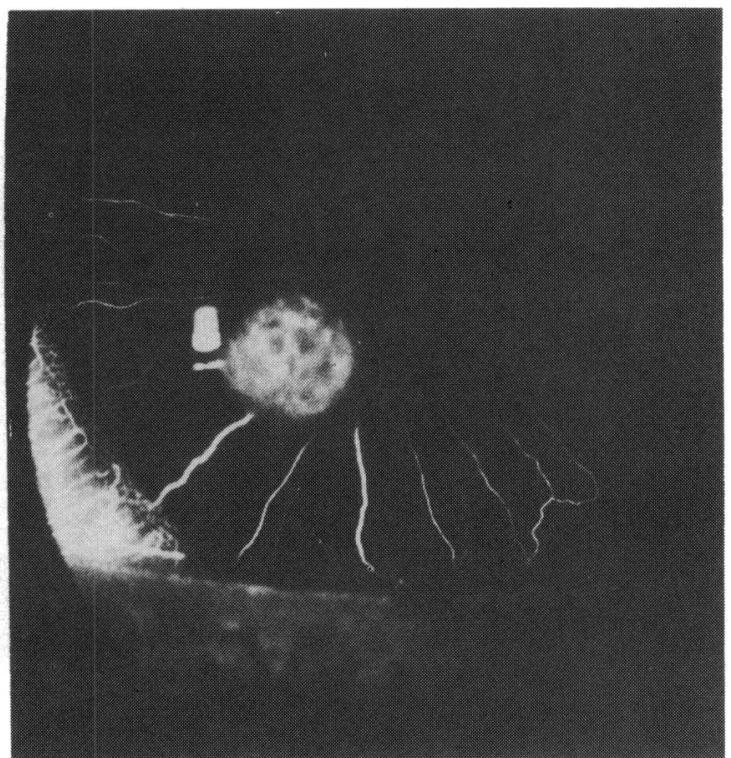

Fig. 3B IFA shows early leakage from a geometric vascular pattern of vessels.

objective, and applicable to all tumours. It addresses the difficulty of having to categorise a borderline angiographic appearance that does not fit one of the previously described patterns, though it is impossible to avoid some degree of subjective assessment - that is, distinguishing between an organised and a disorganised vascular structure.

We failed to differentiate tumours in our inter-

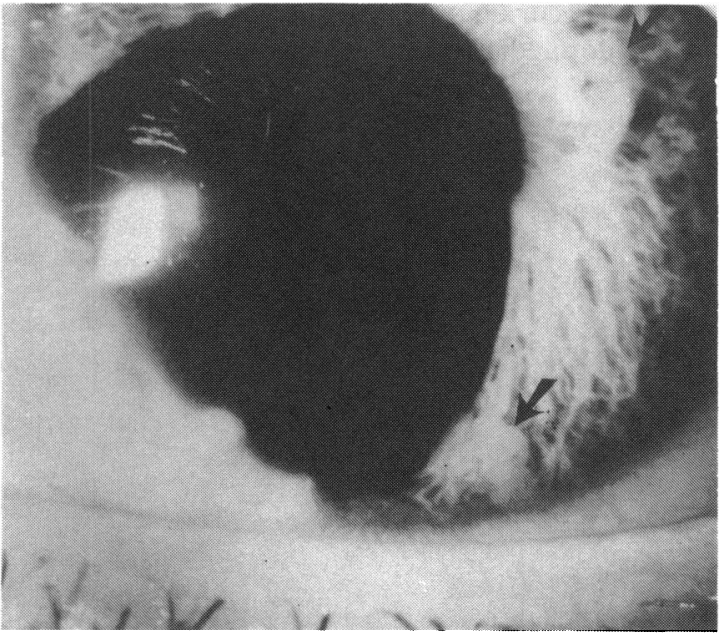

Fig. 3D Clinical appearance of two metastatic lesions on the surface of previously uninvolved iris after broad iridectomy. These were removed with a vitreous cutter, and there has been no further recurrence during the past three years. 


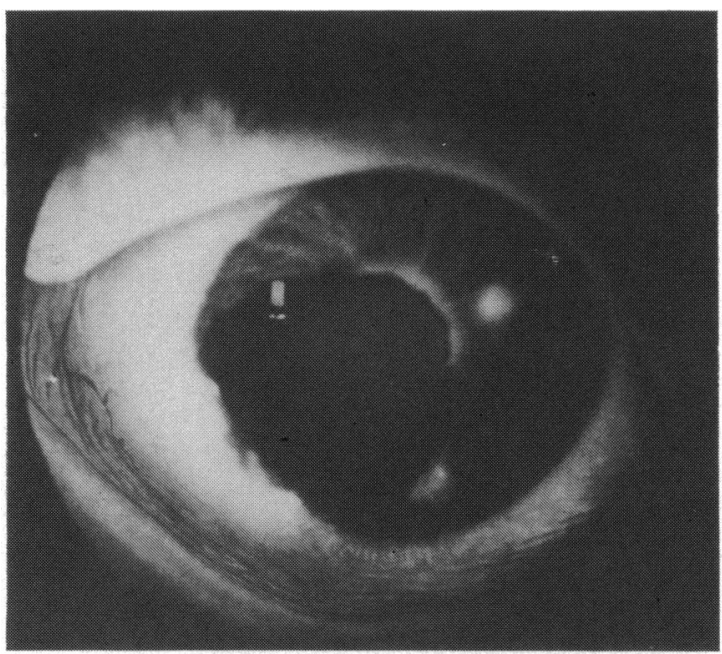

Fig. 3E IFA of the metastatic lesions showing early leakage but no vascularity.

mediate group from malignant tumours on the basis of IFA. We consider that our finding showing that the IFA characteristics of spindle cell naevus with surface plaque and borderline spindle cell naevus overlapped with those of the malignant group justifies our regarding tumours in groups 5 and 6 of the Jacobiec and Silbert classification with suspicion and assigning them to an intermediate group between benign and malignant.

Like other authors ${ }^{4-6}$ we found two features that did not occur in benign tumours-a disorganised vascular pattern and gross late leakage. These features occurred only in 4/7 malignant tumours and in 2/6 intermediate tumours, so although there are no

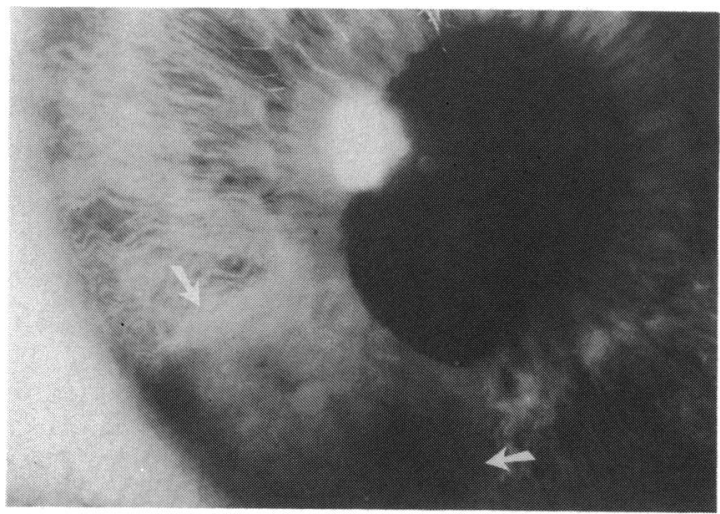

Fig. 4 Case 9. Spindle cell naevus with surface plaque (iris). A: Clinical appearances of a tumour showing slight pigmentation, marked vascularisation involving the angle associated with distortion of the pupil, and ectropion uveae.

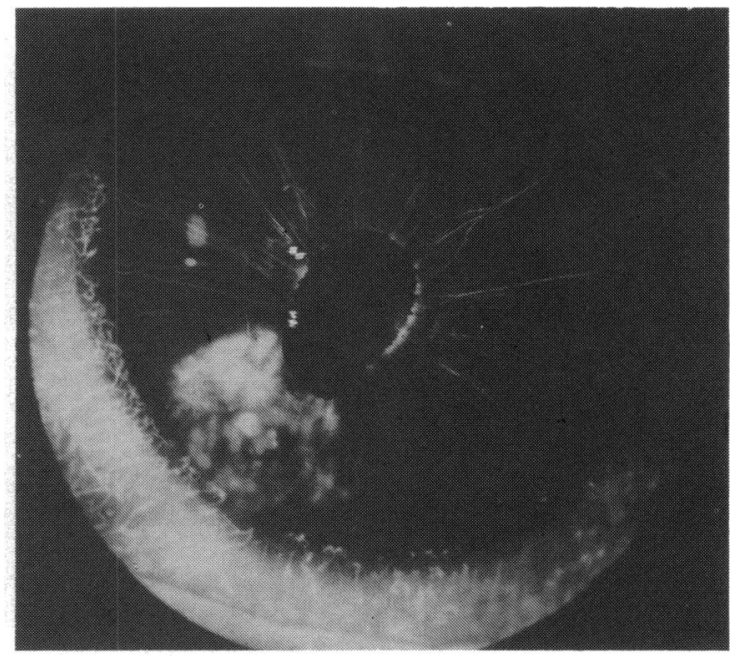

Fig. 4B IFA showing early leakage and a geometric vascular pattern.

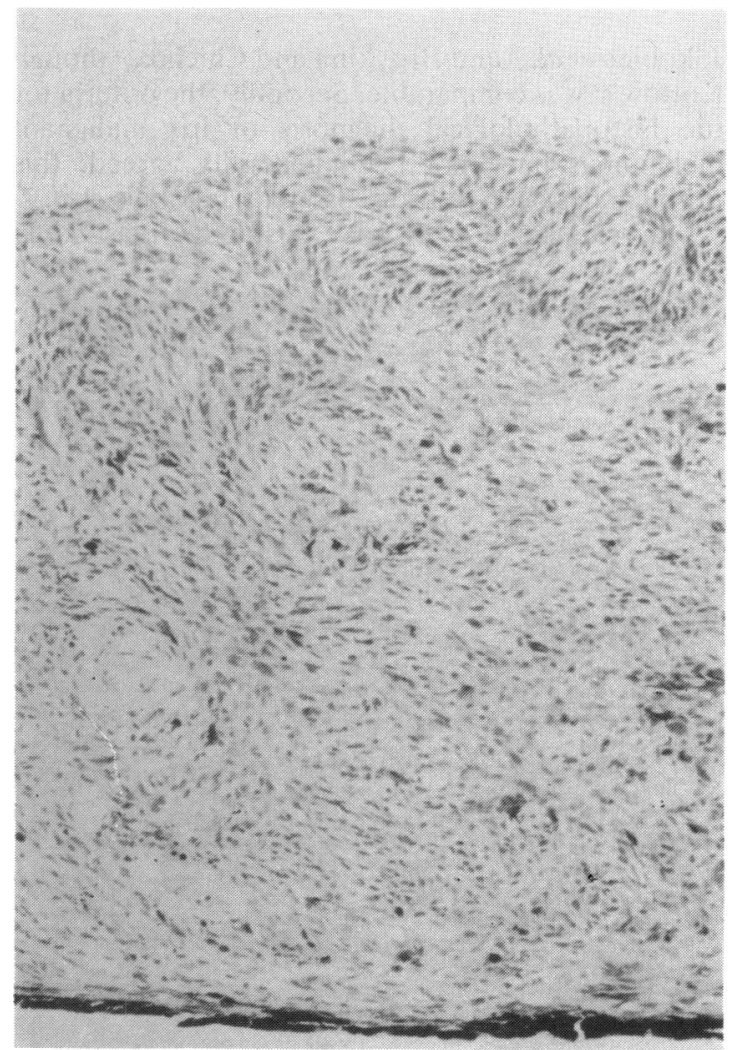

Fig. 4C Histology shows that the stroma is largely replaced by a spindle cell naevus showing a more compact arrangement anteriorly to form a surface plaque. $H$ and $E$, $\times 133$. 


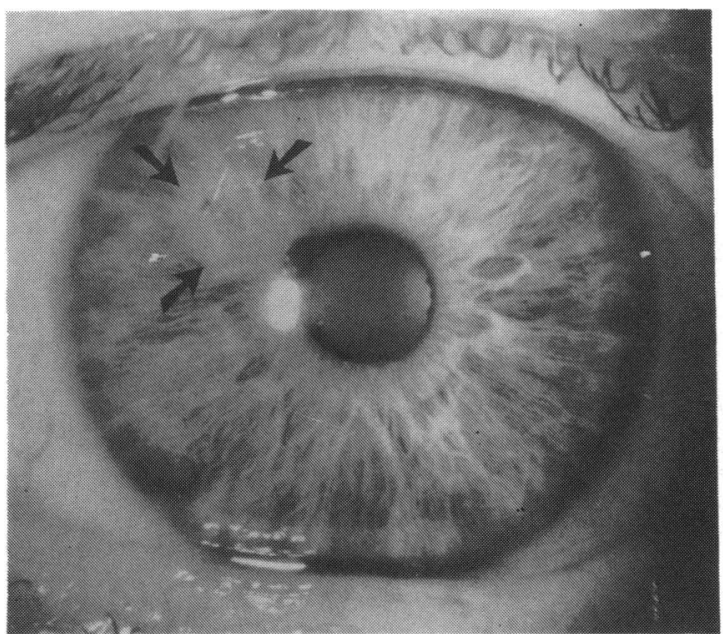

Fig. 5 Case 14. Benign naevus. Clinical appearances unchanged in 66-month follow-up. No biopsy has been done. A: Clinical appearance showing a non-pigmented vascular tumour associated with ectropion uveae.

false positives in our series these findings occur in only about half of the malignant tumours. Early leakage from permeable tumour vessels was found in all groups, although most commonly in malignant and intermediate tumours. It is probably a useful sign of aggressive tumour behaviour provided it is appreciated false positives may occur. The three malignant melanomas of the ciliary body displayed a different pattern from the malignant iris tumours by virtue of their large radial vessels. This is an observation of some importance, since just such a pattern was described in one series as being associated with benign behaviour. ${ }^{+}$

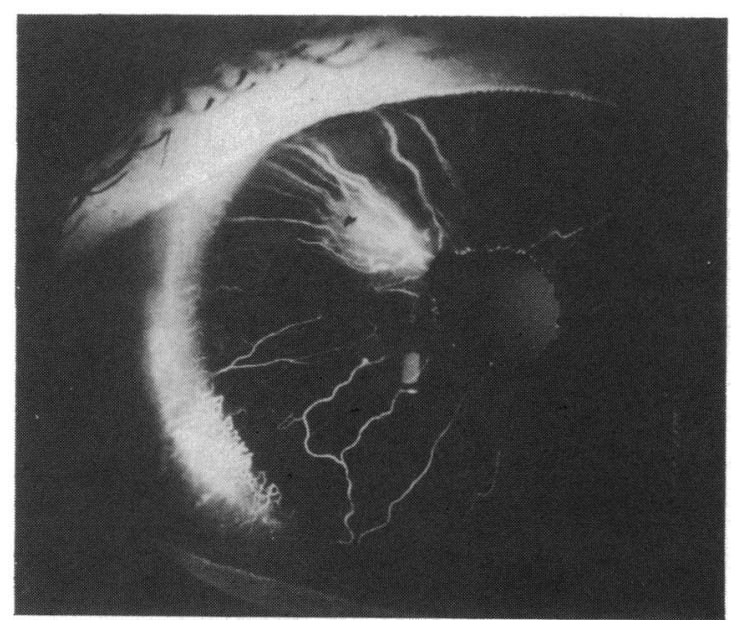

Fig. SB IFA shows early leakage and a geometric vascular pattern.

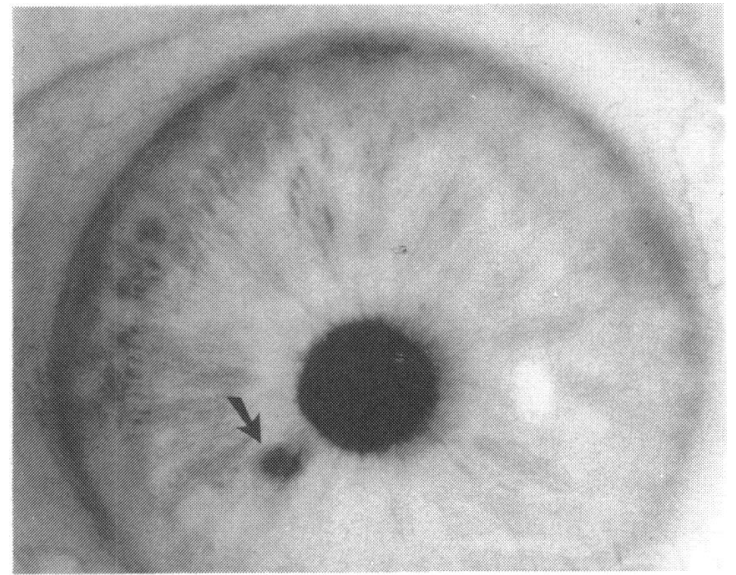

Fig. 6 Case 13. Benign naevus. Clinical appearances unchanged in 90-month follow-up. No biopsy has been done. A: Clinical appearance showing a moderately pigmented tumour associated with iris freckles.

Complete masking was the only feature shown exclusively by benign tumours. This has also been reported in all other series with the exception of one case $^{5}$ in which the histology was said to be malignant, although, unfortunately the histological criteria were not described. We do not consider that the experience of one incompletely substantiated case is sufficient cause to regard complete masking of fluorescence as an unreliable feature of benign tumours. Tumours showing a geometrically organised vasculature were found in all groups, and we cannot agree with Jakobiec et al. ${ }^{4}$ or Brovkina and Chichua $^{6}$ that these are exclusively benign; one other author has recently described similar findings to ours in a further case. ${ }^{9}$

The other fluorescein features observed-

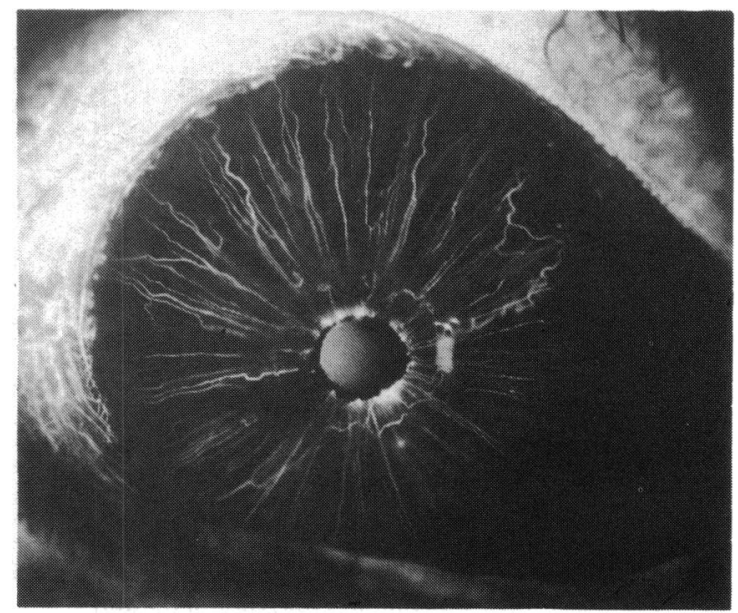

Fig. 6B IFA shows complete masking of fluorescein. 


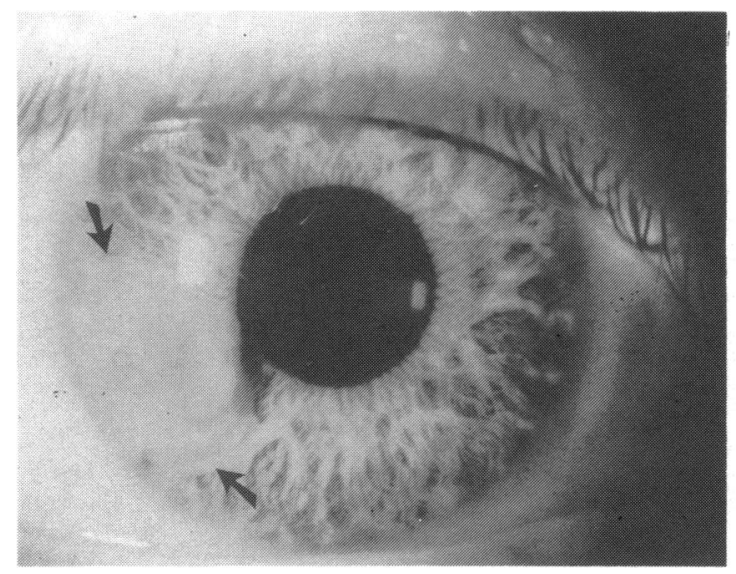

Fig. 7 Case 5. Spindle cell melanoma. A: Clinical appearances showing a markedly vascular non-pigmented tumour involving the angle.

intermediate degrees of masking or leakage, dilatation of radial vessels and the filling of a tumour in different phases-were not associated with any one tumour category but were common to all three groups. We could not substantiate the theoretical prediction that malignant tumours would have vessels that perfused out of phase with the main iris vasculature,${ }^{4}$ as this occurred in all groups.

Of the 11 patients that underwent repeated fluorescein angiography only three proved to be malignant. Although tumour growth was documented, in none of the cases did the fluorescein angiographic categorisation alter despite a follow-up

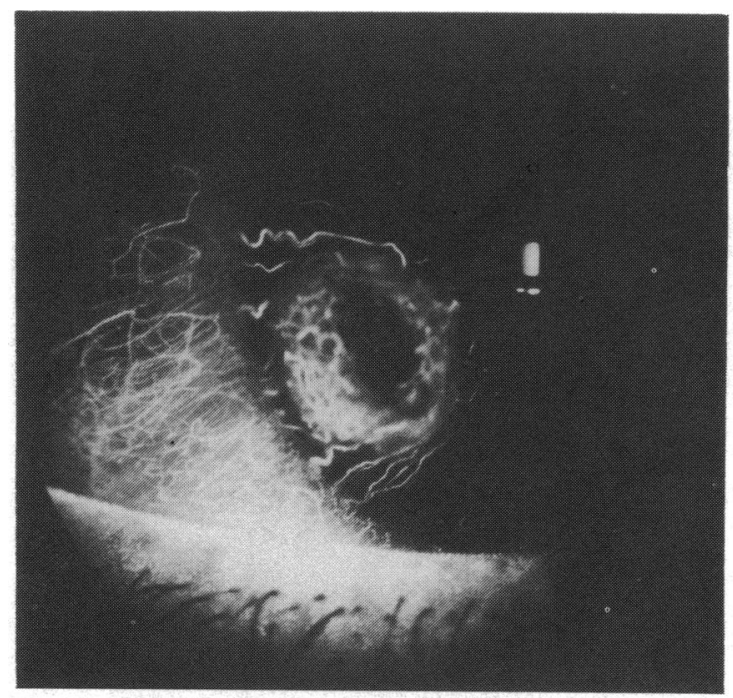

Fig. 7B IFA showing incomplete masking, some late leakage, and a geometric vascular pattern.

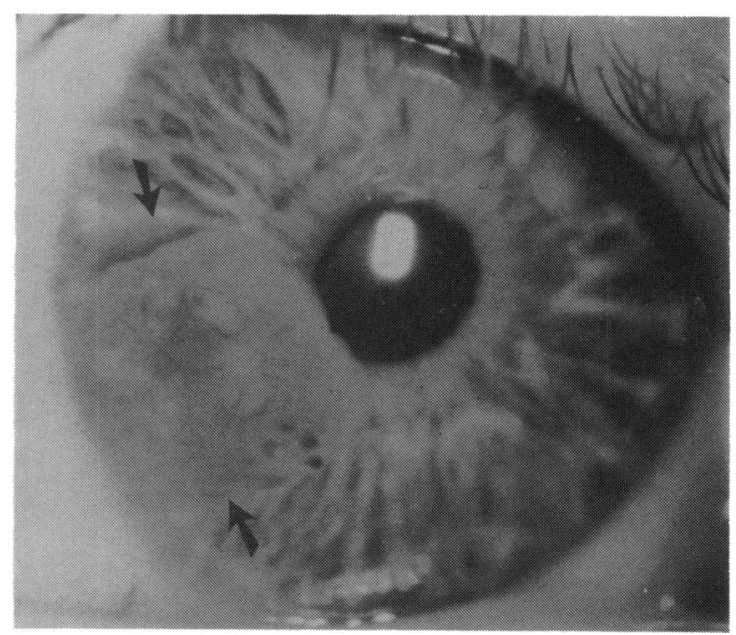

Fig. 7C Clinical appearances 70 months later showing an increase in size that is documented on the IFA shown in $7 D$.

period before surgery of $15-82$ months. This is disappointing in view of the value of IFA in the follow-up of iris tumours that has been predicted, though not reported, by other authors. ${ }^{3-5}$ This failure to demonstrate any IFA change is not surprising, because, although the long history of many of these lesions suggests that malignancies arise in previous naevi, patients probably present only when a change in appearance has occurred-that is, after malignant transformation has taken place.

It is to be expected that exceptions to our present findings will emerge in time. The inability to differentiate tumours of intermediate cytology from those with malignant cytology is a limitation of IFA and

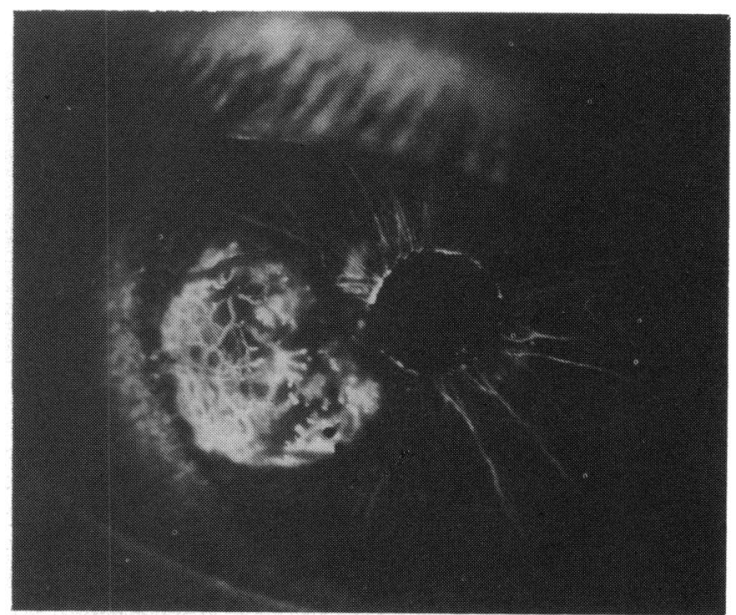

Fig. 7D IFA of 7C showing the increase in size compared with $7 B$. The IFA classification of this tumour had not changed, however. 


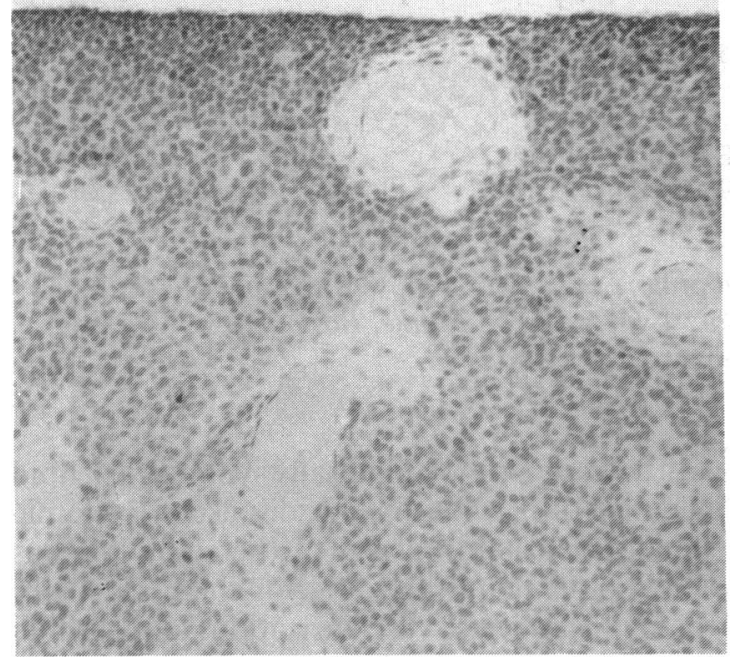

Fig. 7E Histology of part of the anterior surface of the iris showing a uniform spindle cell melanoma with prominent vascularisation. $H$ and $E, \times 127$.

reflects the problems of inferring tumour behaviour from the histology of these lesions. It is also known that benign progressive naevi may cause blindness by local growth and local invasion, although they have no metastatic potential. ${ }^{10}$ The importance of identifying them before they become too large for simple surgical removal is obvious, and unfortunately IFA is unlikely to be able to identify these lesions.

At present the findings of a disorganised vasculature and gross leakage from tumour vessels indicates a tumour of malignant or intermediate cytology that demands careful follow-up. This occurred in $7 / 13$ $(53 \%)$ of such tumours in our series. The only pattern

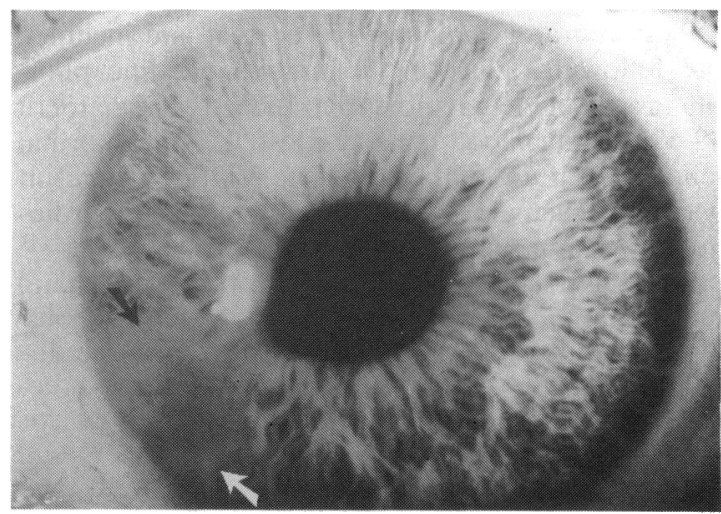

Fig. 8 Case 33. Borderline spindle cell naevus (iris). A: Clinical appearances showing a vascular, moderately pigmented tumour involving the angle and associated with a distorted pupil.

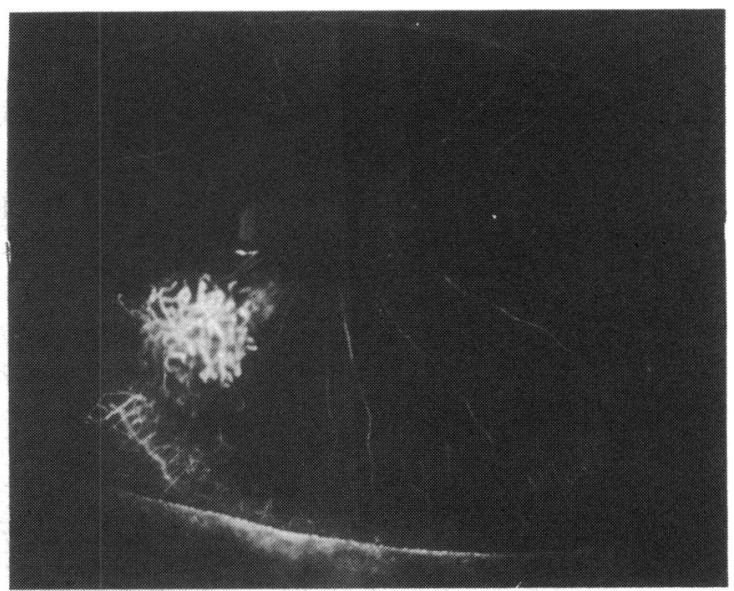

Fig. 8B IFA showing a geometric vascular pattern.

that has been shown by all authors to represent a benign lesion is complete masking of fluorescein without leakage, which was found in $4 / 9$ (44\%) tumours displaying benign behaviour in our series. Although we cannot endorse the assertion that IFA will yield reliable prognostic data in $90 \%$ of cases, ${ }^{4}$ it did give useful information in $11 / 22(50 \%)$ of the cases in our series. Other fluorescein patterns were found not to have enough specificity to give useful information.

We thank the following for their assistance: S M Ford with the fluorescein and colour photography, Peter Clarke and David Powell for statistical advice, F Latif and R Emmett for secretarial help, and the Departments of Medical Illustrations at St Mary's Hospital and the Institute of Ophthalmology for preparing the illustrations.

\section{References}

1 Jakobiec FA, Silbert G. Are most iris melanomas 'really naevi'? Arch Ophthalmol 1981; 99: 2117-32.

2 Noor Sunba MS, Rahi AHS, Morgan G. Tumours of the anterior uvea. 1. Metastasizing malignant melanoma of the iris. Arch Ophthalmol 1980; 98: 82-5.

3 Demeler U. Fluorescence angiographical studies in the diagnosis and follow-up of tumours of the iris and ciliary body. Adv Ophthalmol 1981; 42: 1-17.

4 Jakobiec FA, Depot MJ, Henkind P, Spencer WH. Fluorescein angiographic patterns of iris melanocytic tumours. Arch Ophthalmol 1982; 100: 1288-99.

5 Kottow M. Fluorescein angiographic behaviour of iris masses. Ophthalmologica (Basel) 1977; 174: 217-23.

6 Brovkina AF, Chichua AG. Value of fluorescein iridography in diagnosis of tumours of the iridociliary zone. Br J Ophthalmol 1979; 63: 157-60.

7 Cheng H, Bron A, Easty D. A study of iris masses by fluorescein angiography. Trans Ophthalmol Soc UK 1971; 91: 199-205.

8 Marsh RJ, Ford SM. Cine photography and video recording of anterior segment fluorescein angiography. Br J Ophthalmol 1978; 62: 657-9.

9 Kersten RC, Tse DT, Anderson R. Iris melanoma. Nevus or malignancy? Ophthalmology 1985; 29: 423-33

10 Greer CH. Ocular pathology. 2nd ed. Oxford: Blackwell, 1972.

Accepted for publication 12 March 1987. 